\title{
A LOWER BOUND FOR THE FUNDAMENTAL FREQUENCY OF A CONVEX REGION
}

\author{
M. H. PROTTER ${ }^{1}$
}

\begin{abstract}
A lower bound for the first eigenvalue of the Laplace operator is obtained in terms of the radius of the largest ball which can be inscribed in a convex region in $R^{n}, n>2$.
\end{abstract}

1. Introduction. Recent progress on lower bounds for $\lambda_{1}$, the first eigenvalue of the Laplacian in a region $G$ subject to homogeneous Dirichlet boundary conditions, has shown that $\lambda_{1}$ cannot be arbitrarily small unless the region contains a ball of sufficiently large radius. The first such result for regions in $R^{n}$ was obtained by Hayman [1] and improvements for regions in $R^{2}$ were given by Osserman [3] and for regions in $R^{n}$ by Taylor [7]. A lower bound of the same type for convex regions in $R^{2}$ was found some time ago by Hersch [2] who used a technique which he and the author [6] developed simultaneously.

Hersch showed that for a convex region $G$ in $R^{2}$

$$
\lambda_{1} \geqslant \pi^{2} / 4 \rho^{2},
$$

where $\rho$ is the radius of the largest disk contained in $G$. By means of a lemma of Gale the technique of Hersch extends to regions in $R^{n}, n>3$, with the same inequality (1.1) holding for all values of $n$. Payne and Stakgold [5] established a lower bound for $\lambda_{1}$ for regions $G$ which are not necessarily convex. Their inequality is in terms of $\rho$ and the curvature of the boundary of $G$. For convex domains in $R^{n}$ the result of Payne and Stakgold reduces to (1.1).

In this paper we improve inequality (1.1) by showing that for bounded convex regions $G$ in $R^{n}, n \geqslant 2$, the inequality

$$
\lambda_{1} \geqslant \frac{\pi^{2}}{4}\left(\frac{1}{\rho^{2}}+\frac{n-1}{d^{2}}\right)
$$

holds where $d$ is the supremum of the distance between any two points of $G$. Inequality (1.1) is best possible in the sense that equality is achieved for an infinite strip of width $2 \rho$. As the diameter of $G$ tends to infinity, inequality (1.2) yields the same result, but it is not known if (1.2) is best possible for bounded domains.

In $\$ 2$, inequality (1.2) is established for $n=2$ by a refinement of the techniques developed in [2], [6]. In $\$ 3$ Hersch's result for $R^{2}$ is shown to be valid in $R^{n}, n>3$. This already improves the corresponding result obtained by Osserman [4] and

Received by the editors October 10, 1979.

AMS (MOS) subject classifications (1970). Primary 35J05.

${ }^{1}$ Supported in part by NSF Grant MCS-79-05791. 
matches the result of Payne and Stakgold. In addition, we sketch the method which establishes the stronger result (1.2) for $R^{n}, n>2$.

2. Convex regions in $R^{2}$. Let $D$ be a convex region in $R^{2}$. We denote by $\rho$ the radius of the largest circle which can be inscribed in $D$ and by $d$ the diameter of $D$, i.e., the supremum of the distance between any two points of $D$. We consider the eigenvalue problem

$$
\Delta u+\lambda u=0 \text { in } D, \quad u=0 \text { on } \partial D .
$$

THEOREM 1. Let $\lambda_{1}$ be the first eigenvalue of (2.1). Then

$$
\lambda_{1}>\frac{\pi^{2}}{4}\left(\frac{1}{\rho^{2}}+\frac{1}{d^{2}}\right) .
$$

Proof. We consider three cases.

Case 1. The domain $D$ is contained in a strip of width $2 \rho$. In this case, $D$ is contained in a rectangle of width $2 \rho$ and length not exceeding $d$. By the inclusion principle it follows that $\lambda_{1} \geqslant \pi^{2} / 4 \rho^{2}+\pi^{2} / d^{2}$ which is stronger than (2.2).

If $D$ does not lie in a strip of width $2 \rho$, then the disk $K$ of radius $\rho$ contained in $D$ must have at least three points of contact with $\partial D$. It is not difficult to see that the set $\partial K \cap \partial D$ cannot be contained in a semicircular arc of $K$. Denote by $T$ the triangle which contains $D$ and whose sides touch $\partial K \cap \partial D$ at three or more points. Then the first eigenvalue for the problem

$$
\Delta u+\lambda u=0 \quad \text { in } T, \quad u=0 \text { on } \partial T
$$

is a lower bound for $\lambda_{1}$. We shall use the method of "effectless cutting" described by Hersch [2] to obtain a lower bound for the first eigenvalue of problem (2.3).

Case 2. The triangle $T$ has all acute angles. Let $A_{1}, A_{2}, A_{3}$ be the vertices of $T$ and choose coordinate axes so that $\overline{A_{1} A_{2}}$ is situated along the $x$ axis. Through the center $C$ of the disk $K$ construct three line segments parallel to the sides of $T$. Let $C B_{1}, C B_{2}, C B_{3}$ denote these segments where $B_{1}, B_{2}, B_{3}$ lie on $\overline{A_{1} A_{3}}, \overline{A_{1} A_{2}}$, and $\overline{A_{2} A_{3}}$, respectively. The triangular $T$ is divided into three quadrilaterals $Q_{1}, Q_{2}, Q_{3}$ with vertices $A_{1} B_{1} C B_{2}, A_{2} B_{2} C B_{3}, A_{3} B_{3} C B_{1}$, respectively. We consider the first eigenvalue for the problem

$$
\begin{aligned}
\Delta u+\lambda u=0 & \text { in } Q_{1}, \\
u=0 & \text { on } \overline{A_{1} B_{1}} \cup \overline{A_{1} B_{2}}, \\
\partial u / \partial n=0 & \text { on } \overline{B_{1} C} \cup \overline{C B_{2}} .
\end{aligned}
$$

The first eigenvalue for problem (2.4) together with the analogous problems for the quadrilaterals $Q_{2}$ and $Q_{3}$ yield three first eigenvalues, the minimum of which is a lower bound for $T$ and hence for $D$. We now show that (2.2) is a lower bound for (2.4).

Let $p(x, y), q(x, y)$ be bounded $C^{1}$ functions defined in $Q_{1}$. It is known [2], [6] that a lower bound for $\lambda_{1}\left(Q_{1}\right)$ is given by

$$
\lambda_{1}>\inf _{(x, y) \in Q_{1}}\left[p_{x}+q_{y}-p^{2}-q^{2}\right]
$$


provided that

$$
p d y-q d x<0 \text { on } \overrightarrow{B_{2} C} \cup \overrightarrow{C B_{1}} .
$$

Since $d y=0$ and $d x<0$ on $\overrightarrow{C B_{1}}$, condition (2.6) states that $q<0$ on $\overrightarrow{C B_{1}}$. Also, we observe that on the directed segment $\overrightarrow{B_{2} C}$ we have $d y>0, d x<0$. Hence (2.6) holds if $p<0, q \leqslant 0$ on $\overrightarrow{B_{2} C}$. Suppose first that length $\left(\overline{A_{1} B_{2}}\right)<d$. Then for sufficiently small $\varepsilon$ we set

$$
p=-\frac{\pi}{2 d} \cot \frac{\pi}{2 d}\left(\frac{x}{1+\varepsilon}+\varepsilon\right), \quad q=-\frac{\pi}{2 \rho} \cot \frac{\pi}{2 \rho}\left(\frac{y}{1+\varepsilon}+\varepsilon\right) .
$$

Then (2.6) is satisfied and substitution of (2.7) into (2.5) yields the lower bound (2.2) as $\varepsilon \rightarrow 0$. If length $\left(\overline{A_{1} B_{2}}\right)>d$, the quadrilateral $Q_{1}$ may be replaced by a smaller polygon which yields the same bound (2.2). To see this, we consider two vertical lines which are lines of support for $D$ and are distance at most $d$ from each other. The portion of these lines which intersect $Q_{1}$, denoted $l_{1}$ and $l_{2}$, together with the portion of $Q_{1}$ between these lines form a polygon. The boundary condition $u=0$ is imposed on $l_{1} \cup l_{2}$ and the previous boundary conditions are imposed on the remaining sides of this truncated region. It is clear that the functions (2.7) satisfy the appropriate conditions and yield the same bound (2.2).

Case 3. The triangle $T$ has one angle, say $A_{3}$, larger than $\pi / 2$. Since the trapezoid $Q_{3}$ does not have two acute base angles, the argument in Case 2 is not applicable. However, a lower bound for $T$ can be obtained by dividing $T$ into two parts by a line segment $\overline{E F}$ parallel to $\overline{A_{1} A_{2}}$ and at a distance $\tau_{1}$ from it. Then the method of effectless cutting is applied to the triangle $E F A_{3}$ and the trapezoid $A_{1} A_{2} F E$. The first eigenvalue problem for the trapezoid with Dirichlet data on $\overline{E A_{1}} \cup \overline{A_{1} A_{2}} \cup \overline{A_{2} F}$ and with Neumann data on $\overline{E F}$ has the lower bound

$$
\pi^{2}\left(1 / 4 \tau_{1}^{2}+1 / d^{2}\right)
$$

A lower bound for the first eigenvalue problem for the triangle $E F A_{3}$ with Dirichlet data on $\overline{F A_{3}} \cup \overline{A_{3} E}$ and with Neumann data on $\overline{E F}$ is

$$
\pi^{2}\left(1 / 4 \tau_{2}^{2}+1 / \sigma^{2}\right)
$$

where $\sigma$ is the length of $\overline{E F}$ and $\tau_{2}$ is the length of the perpendicular from $A$ to $\overline{E F}$.

Now it is not difficult to show that $\tau_{1}$ and $\tau_{2}$ can be chosen so that (2.8) and (2.9) are larger than (2.2). We give the details for the case where $\overline{A_{1} A_{3}}=\overline{A_{2} A_{3}}$, the general case being similar. Let the angle $A_{3}$ be denoted by $2 \theta$, in which case we find that $\tau_{1}+\tau_{2}=\rho(1+\csc \theta)$. The requirement that (2.8) and (2.9) exceed (2.2) is

$$
\frac{1}{\tau_{1}^{2}} \geqslant \frac{1}{\rho^{2}}\left[\frac{4(1+\sin \theta)^{2}-3 \cos ^{2} \theta}{4(1+\sin \theta)^{2}}\right], \quad \frac{1}{\tau_{2}^{2}} \geqslant \frac{\sin ^{2} \theta}{\rho^{2}}\left[\frac{4(1+\sin \theta)^{2}+\cos ^{2} \theta}{4(1+\sin \theta)^{2}}\right] \text {. }
$$

We choose $\tau_{1}=\alpha \rho(1+\csc \theta), \tau_{2}=\beta \rho(1+\csc \theta)$ with $\alpha(\theta), \beta(\theta)$ positive and $\alpha+\beta \geqslant 1$. It may be verified that for $\pi / 4<\theta \leqslant \pi / 2$, the inequality

$$
\frac{2}{\left[4(1+\sin \theta)^{2}+\cos ^{2} \theta\right]^{1 / 2}}+\frac{2 \sin \theta}{\left[4(1+\sin \theta)^{2}-3 \cos ^{2} \theta\right]^{1 / 2}}>1
$$


holds. Hence $\alpha(\theta), \beta(\theta)$ with the desired properties can be found.

3. Convex regions in $R^{n}$. The method employed in $\$ 2$ can be extended to yield a lower bound for the first eigenvalue for bounded convex regions in $R^{n}, n>3$. The result of Hersch in $R^{2}$ has a straightforward generalization if use is made of the following lemma of David Gale. ${ }^{2}$

LEMMA. Let $G$ be a bounded convex region in $R^{n}$ and suppose $\rho$ is the radius of the largest ball $B$ which lies in $G$. Then for some integer $k$, with $2<k<n+1$, there is a polyhedral domain $H$ consisting of the intersection of $k$ half-spaces which contains $G$ and is such that $H$ contains no ball of radius larger than $\rho$.

To extend Hersch's method, we first consider the problem of finding a lower bound for the first eigenvalue of the Laplacian for a class of pyramidal domains subject to mixed boundary conditions. Let $A$ be an $(n-1)$-dimensional convex polyhedron lying in a hyperplane $H_{0}$. Suppose $P \notin H_{0}$ is such that the perpendicular from $P$ to $H_{0}$ intersects $A$. We denote by $R$ the pyramid in $R^{n}$ formed as follows: construct the line segments from $P$ to the vertices of $A$. Then $R$ is the convex hull of $A$ and the line segments so constructed. We observe that $A$ and therefore $R$ may be unbounded.

We consider the eigenvalue problem

$$
\begin{aligned}
& \Delta u+\lambda u=0 \text { in } R, \\
& u=0 \text { on } A \text {, } \\
& \partial u / \partial n=0 \text { on } \partial R-A \text {. }
\end{aligned}
$$

The method described in [2], [6] is applicable for obtaining a lower bound for the first eigenvalue of problem (3.1). Let $x_{1}$ be the coordinate direction perpendicular to $A$ with $H_{0}$ the hyperplane $x_{1}=0$. We denote by $\rho$ the distance from $P$ to $A$. If $p\left(x_{1}\right)$ is an appropriate trial function then a lower bound for problem (3.1) is given by

$$
\lambda_{1} \geqslant p_{x_{1}}-p^{2} \text {. }
$$

Since the exterior normal at each point of the boundary $\partial R-A$ makes an acute angle with the $x_{1}$-direction, the choice

$$
p\left(x_{1}\right)=-\frac{\pi}{2 \rho} \cot \frac{\pi}{2 \rho} x_{1}
$$

yields the lower bound $\lambda_{1} \geqslant \pi^{2} / 4 \rho^{2}$.

Let $G$ be an arbitrary bounded convex set in $R^{n}$ and suppose that the ball $B$ with center at $P$ and radius $\rho$ is the largest which can be inscribed in $G$. According to the above lemma there is a polyhedral domain $H$ which has the same ball $B$ as its maximum inscribed ball. Now we employ the method of effectless cutting. We construct the pyramidal domains $R_{1}, R_{2}, \ldots, R_{k}$ using in each case $P$ as the vertex and successively the $(n-1)$-dimensional faces of $H$. Then a lower bound for the first eigenvalue for $G$ is given by finding the minimum of the first eigenvalues of $R_{1}, R_{2}, \ldots, R_{k}$ corresponding to problem (3.1). However, each of these problems

\footnotetext{
${ }^{2}$ Private communication.
} 
yields a lower bound $\lambda_{1} \geqslant \pi^{2} / 4 \rho^{2}$. We observe that this result is an improvement of the value $1 / 4 \rho^{2}$ obtained by Osserman [4] and the same as that of Payne and Stakgold [5].

By extending the methods described in $\$ 2$, it is possible to obtain a higher lower bound in terms of $\rho$, the radius of the largest inscribed ball, and $d$, the diameter of G.

TheOREM 2. Let $G$ be a bounded convex set in $R^{n}$. Then the first eigenvalue $\lambda_{1}$ for the problem

$$
\Delta u+\lambda u=0 \quad \text { in } G, \quad u=0 \text { on } \partial G
$$

has the lower bound

$$
\lambda_{1} \geqslant \frac{\pi^{2}}{4}\left(\frac{1}{\rho^{2}}+\frac{n-1}{d^{2}}\right) .
$$

Proof. According to the lemma of Gale, there is a polyhedral domain $H$ which has the ball $B$ of radius $\rho$ as its largest inscribed ball. We again use the method of effectless cutting, and we describe the situation for $n=3$, the case of higher dimensions being similar. Denote the center of the ball $B$ by $P$ and suppose first that $H$ is a tetrahedron. Through $P$ we pass planes parallel to the faces of $H$. These planes together with the faces of $H$ decompose $H$ into four polyhedra $Q_{1}, \ldots, Q_{4}$. Each polyhedron $Q_{i}$ contains one vertex of $H$ and has six faces. Three of the six will be portions of $\partial H$ and the other three will be portions of the interior planes passing through $P$. We denote by $S_{i j}, T_{i j}, i=1, \ldots, 4, j=1,2,3$, the portions of the faces of $Q_{i}$ belonging to $\partial H$ and to the interior surfaces, respectively. We consider the problems

$$
\begin{aligned}
\Delta u+\lambda u=0 & \text { in } Q_{i} \\
u=0 & \text { on } \bigcup_{j} S_{i j}, \\
\partial u / \partial n=0 & \text { on } \bigcup_{j} T_{i j},
\end{aligned}
$$

and we obtain lower bounds for each of these problems. One of the surfaces $S_{i j}$ will be parallel to and at distance $\rho$ from the corresponding surface $T_{i j}$. The remaining pairs of surfaces will be at most at distance $d$ apart. Hence problem (3.5) has the lower bound (3.4).

If the polyhedron $H$ is unbounded in some direction, then the inclusion principle provides the estimate $\pi^{2} / 4 d^{2}$ in this direction and the argument in the remaining directions is unchanged. If in any direction the projection of the polyhedral angle in that direction exceeds $\pi / 2$, the argument in the two-dimensional case described in $\$ 2$ may be applied.

\section{BIBLIOGRAPHY}

1. W. K. Hayman, Some bounds for principal frequency, Applicable Anal. 7 (1978), 247-254.

2. J. Hersch, Sur la fréquence fondamentale d'une membrane vibrante; évaluation par défaut et principe de maximum, J. Math. Phys. Appl. 11 (1960), 387-413. 
3. R. Osserman, A note on Hayman's theorem on the bass note of a drum, Comment. Math. Helv. 52 (1977), 545-555.

4. B_ Bonneson-style isoperimetric inequalities, Amer. Math. Monthly 86 (1979), 1-29.

5. L. E. Payne and I. Stakgold, On the mean value of the fundamental mode in the fixed membrane problem, Applicable Anal. 3 (1973), 295-303.

6. M. H. Protter, Lower bounds for the first eigenvalue of elliptic equations, Ann. of Math. (2) 71 (1960), 423-444.

7. M. E. Taylor, Estimate on the fundamental frequency of a drum, Duke Math. J. 46 (1979), $447-453$.

Department of Mathematics, University of California, Berkeley, California 94720 